\title{
Monte Carlo track structure simulation in studies of biological effects induced by accelerated charged particles in the central nervous system
}

\author{
Munkhbaatar Batmunkh ${ }^{1, *}$, Lkhagvaa Bayarchimeg ${ }^{1,2}$, Aleksandr N. Bugay ${ }^{1}$, and \\ Oidov Lkhagva ${ }^{3}$ \\ ${ }^{1}$ Laboratory of Radiation Biology, Joint Institute for Nuclear Research, Dubna, 141980, Russia \\ ${ }^{2}$ Institute of Physics and Technology, Mongolian Academy of Science, Ulaanbaatar, 13330, Mongolia \\ ${ }^{3}$ Division of Natural Sciences, National University of Mongolia, Ulaanbaatar, 210646, Mongolia
}

\begin{abstract}
Simulating the biological damage induced by charged particles trajectories (tracks) in the central nervous system (CNS) at different levels of its organization (molecular, cellular, and tissue) is a challenge of modern radiobiology studies. According to the recent experimental studies at particle accelerators, the most radiation-sensitive area of the CNS is the hippocampus. In this regards, the development of measurement-based Monte Carlo simulation of radiation-induced alterations in the hippocampus is of great interest to understand the radiobiological effects on the CNS. The present work investigates the influence of charged particles on the hippocampal cells of the rat brain using the Geant4 Monte Carlo radiation transport code. The applied computer simulation provides a method to simulate physics processes and chemical reactions in the developed model of the rat hippocampus, which contains different types of neural cells - pyramidal cells, mature and immature granular cells, mossy cells, and neural stem cells. The distribution of stochastic energy depositions has been obtained and analyzed in critical structures of the hippocampal neurons after irradiation with $600 \mathrm{MeV} / \mathrm{u}$ iron particles. The computed energy deposition in irradiated hippocampal neurons following a track of iron ion suggests that most of the energy is accumulated by granular cells. The obtained quantities at the level of molecular targets also assume that NMDA and GABA receptors belong to the most probable targets in the irradiated neural cells.
\end{abstract}

\section{Introduction}

The track structure of accelerated charged particles with high linear energy transfer (LET) produces greatly different patterns of energy deposition compared to ionizing electromagnetic radiation (X-and $\gamma$-rays) when they penetrate into the brain cells (neurons). By means of Monte Carlo approach, it is possible to simulate the stochastic nature of energy deposition and water radiolysis in the tracks of charged particles [1]. An important aspect in this simulation is the geometrical description of the complicated neuron morphology and populations. Most neurons have soma or cell body, axons, complex dendritic trees with multiple branches, and dendritic spines with ionotropic receptors which are the substrates for synaptic transmission.

\footnotetext{
*e-mail: batmunkh@jinr.ru
} 
Known effects of charged particles traversing the structures of the CNS include degradation of dendrite tree morphology $[2,3]$ and disturbance of electrochemical processes governing synaptic transmission $[4,5]$. This earliest radiation injury to individual neurons results in long-term effects including various impairments of behavior, memory and other cognitive deficits $[6,7]$. The analysis of recent experimental studies at particle accelerators with energetic heavy ions suggests that the hippocampus is one of the most sensitive regions of the CNS under irradiation [5, 8]. On the other hand, rodent hippocampal neurons are among the most intensively studied neural systems. In the rat hippocampus, there are two main types of principal neurons: population of 1.2 million granule cells in dentate gyrus (DG) region and population of 614000 pyramidal cells in cornu ammonis (CA3/CA2/CA1) region, respectively [9].

In the present work we consider the model of the rat hippocampus to establish a basis to simulate realistic large-scale neural networks followed by energy deposition in particle tracks. The track structures of energetic heavy ions and their secondary particles produced within critical targets of hippocampal neurons have been scored and analyzed.

\section{Materials and methods}

We have performed a Monte Carlo radiation transport simulation (Geant4.10.4 code [10]) to investigate the influence of heavy charged particles on the rat hippocampus from molecules to neuron populations. In particular, the Geant4-DNA [11] processes are used inside the critical neuron structures and allow to transport the charged particles as well as secondary electrons down to very low energies $(7.4 \mathrm{eV})$. The simulation medium was filled with water, which constitutes an approximation for biological material within complex geometries as detailed below.

\subsection{Modeling of individual neurons with spines and receptors}

Sample 3D geometries of individual CA1 pyramidal neurons with spines and receptors are illustrated in Fig. 1. A single CA1 pyramidal neuron is simulated with $1.5 \cdot 10^{4}$ spines and $1.9 \cdot 10^{6}$ receptors, respectively. The complex morphology of dendritic trees was constructed from the microscopy-based data in the Claiborne archive at the neuromorpho.org repository. Spines and three types of receptors (NMDA, AMPA and GABA) are randomly distributed along the dendrites and within the post-synaptic density (PSD) without overlaps. The detailed geometrical description of spines head and neck, and receptors as molecular targets has been described in detail elsewhere [12-14]. A full description of the neuron geometry implementation in Geant4 is already available in our previously published works and Geant4 application [15-17].

\subsection{Modeling of the rat hippocampus irradiation}

To overcome computational difficulties in neuro-radiobiological studies, we have developed simplified neuron models with properties equivalent to realistic neuron morphology $[12,18]$. Using the simplified neuron models, we have simulated an 1:100 scale model of the rat hippocampus. The model illustrated in Fig. 2 contains populations of 3840 CA1 pyramidal neurons, 2401 CA3/CA2 pyramidal neurons, 11960 DG granule cells, 496 mossy cells in hilus (HMC), 560 DG immature neurons (IMN) and 110 neural stem cells (NSC) in the subgranular zone. The neurons have been simulated in the hippocampal regions within the water box of overall dimensions of $4608 \times 7106 \times 1000-\mu \mathrm{m}$ [9]. The distributions of energy deposition events were computed for track of ${ }^{56} \mathrm{Fe}$ particles with energy of $600 \mathrm{MeV} / \mathrm{u}$. These 


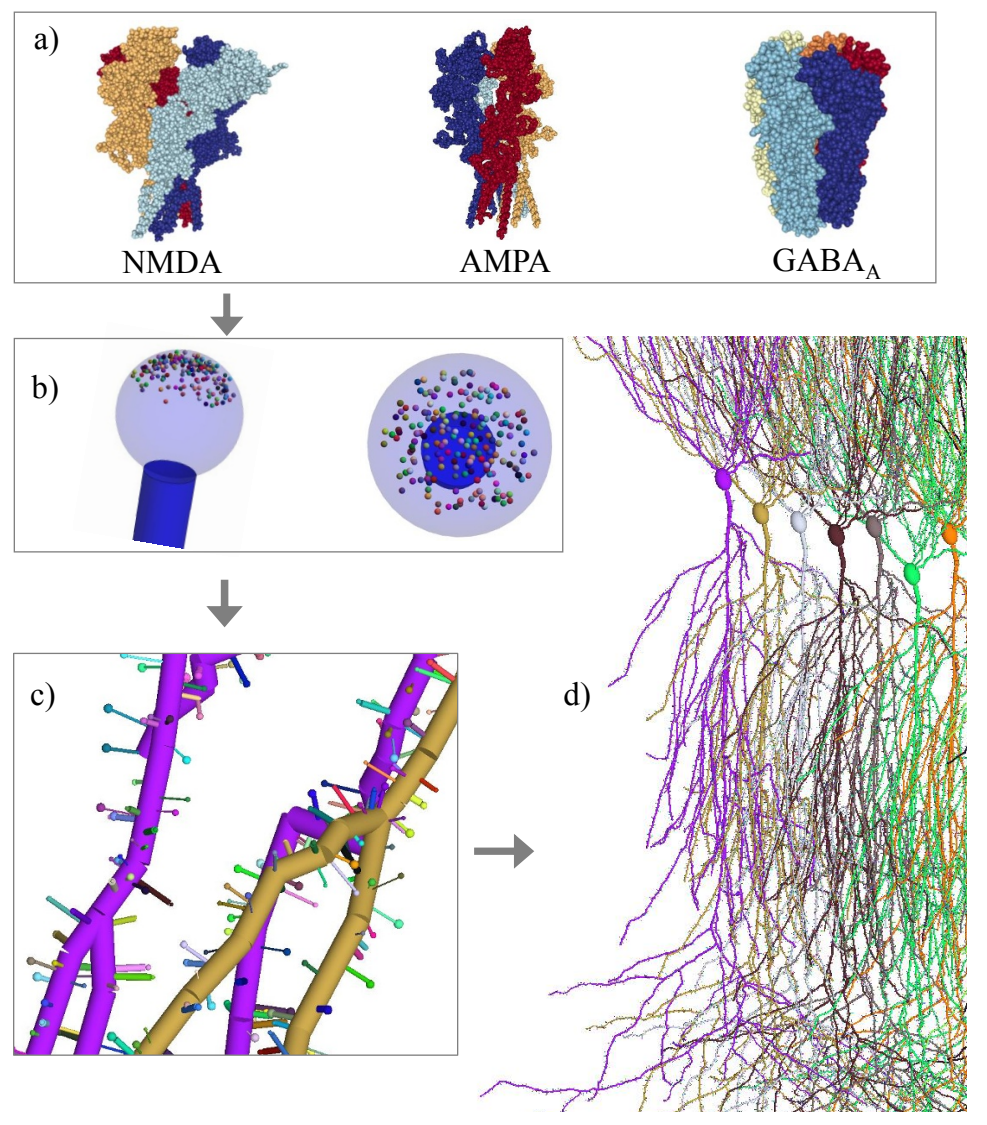

Figure 1. Organization of ionotropic receptors and spines on dendritic trees of neural cells: a) An atomistic model of NR1a/2b NMDA, GluA2/3 AMPA and $\mathrm{GABA}_{\mathrm{A}}$ receptor ( $\beta 3$ homopentamer). Different colors are used to represent four and five subunits of glutamate (NMDA, AMPA) and $\mathrm{GABA}_{\mathrm{A}}$ receptor. b) A head of dendritic spine with receptors randomly placed within the PSD. c) A dendritic segment of individual neurons with spines and receptors. d) A snapshot of CA1 pyramidal neuron network in the model of the rat hippocampus

particles are present in the spectra of galactic cosmic rays and have several times higher biological efficiency than for other particle beams. Particles were generated from the box edges and randomly directed inside the box, containing the hippocampus, and then the energy depositions in track structures of primary and secondary particles were computed for each type of the irradiated neurons.

\section{Results and discussion}

\subsection{Memory usage during the simulation}

Simulating a complex geometry of biological targets with multiple elements could naively require a huge amount of physical memory of modern computers. In contrast to Monte Carlo track simulation in the Geant4-DNA environment would take terabytes of RAM for targets with a size of hundreds and thousands of cells and heavy charged particles producing 
a)

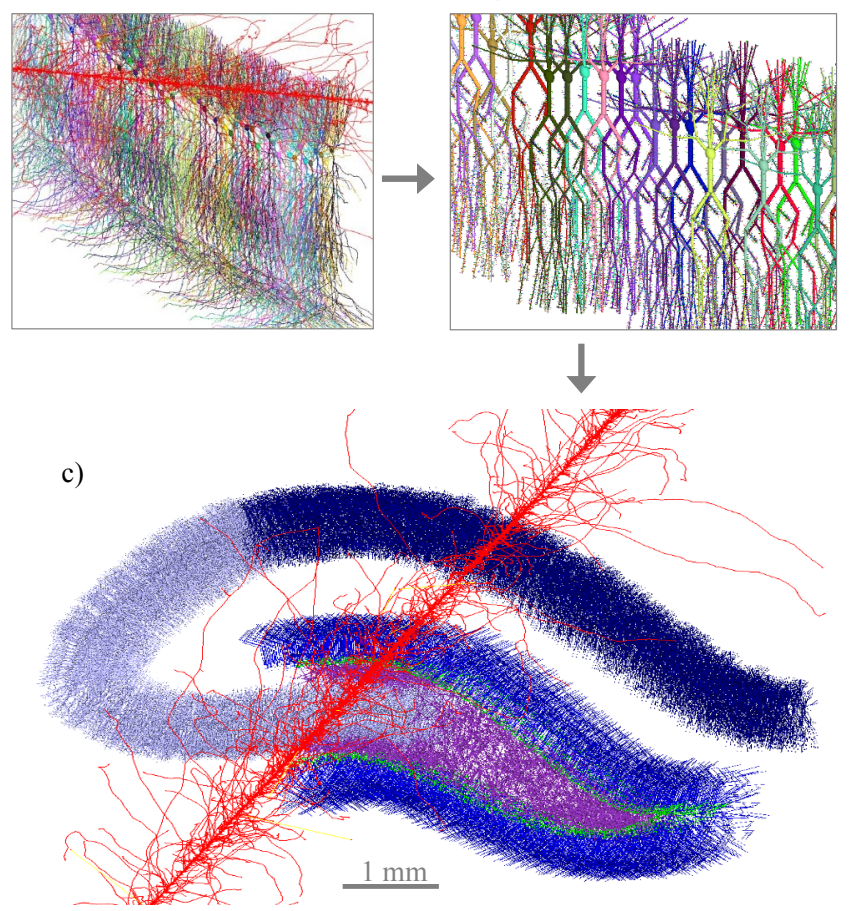

Figure 2. A sample

three-dimensional

representation of different

neurons in the rat

hippocampus which are

traversed by single track of

$600 \mathrm{MeV} / \mathrm{u}$ iron ion. An

example of realistic (a) and

simplified (b) networks of individual pyramidal neurons in the CA1 region. The main panel (c) shows the scaled model of hippocampal neurons (dorsal view); the DG granule cells are highlighted in blue, neurons in the subgranular zone - green, mossy fibers in the hilus purple, CA1 pyramidal neurons - dark blue, and CA3/CA2 pyramidal neurons - light blue. The energy deposition in track structure of iron ion is given in red

a great number of physical and chemical events. The total amount of the memory used for simulations with different models of CA1 pyramidal neurons at the level of single cells and populations is shown in Fig. 3. The amount of the used memory increases with the number of neurons for both the realistic and simplified geometries. However, the realistic geometry results in several times higher memory consumption at the level of neural networks. According to the simulation results $[12,18]$, the differences between realistic and simplified neuron model have been found to be very small in terms of total energy depositions in soma, dendritic tree, and the spines distributed along the dendrites.

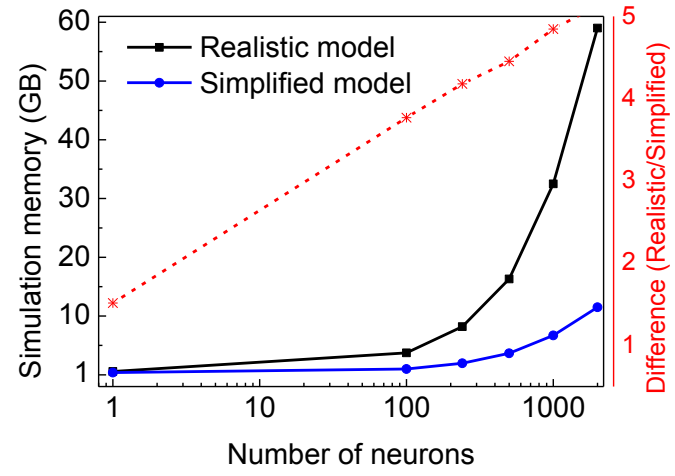

Figure 3. Total amount of the physical memory used for constructing geometry in neural networks composed of realistic and simplified models of pyramidal neurons in the Geant4 Monte Carlo simulation. Red curves show ratio between the realistic and simplified models

\subsection{Evaluation of energy deposit in possible targets of hippocampus}

The mean number of irradiated neurons in the rat hippocampus by a single track of 600 $\mathrm{MeV} / \mathrm{u}$ iron particle is plotted in Fig. 4. It is shown that the largest number of irradiated 
neurons has been found in the DG region of hippocampus, because a large number of DG granule cells are located in the DG region. On the other hand, the amount of energy deposition in neural stem cells is much smaller than other types of hippocapal neurons (see Fig 4b). However, these numbers could be of great importance, because proliferating neural stem cells are the most radiosensitive in contrast to other more abundant neuron types throughout the hippocampus.
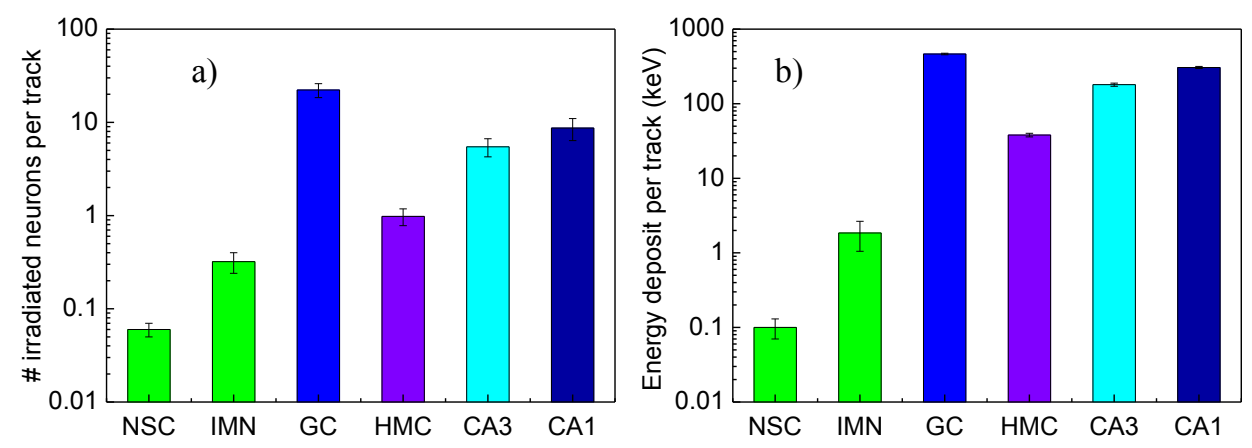

Figure 4. Mean number of irradiated neurons in different regions of the rat hippocampus following the exposure to a single iron ion with energy of $600 \mathrm{MeV} / \mathrm{u}$ (a). After irradiation, energy deposition in hippocampal neurons per track of iron ion (b)

Fig. 5 presents a detailed analysis of energy depositions within the geometry of the critical neuron structures after irradiation with $600 \mathrm{MeV} / \mathrm{u}$ iron ion. In the case of CA1 pyramidal neuron (Fig 5a), it appears that more than $80 \%$ of the total energy deposition occurred in dendrites, and energy in spines $(8.3 \%)$ is a little bit higher than in soma $(7.9 \%)$. In the case of DG granule cells, the largest portion of direct hits by energy deposition were obtained in dendrites and soma compared to spines as estimated previously $[12,17]$. For receptors, $\mathrm{GABA}_{\mathrm{A}}$ has received the largest amount of energy deposition with more than $1 \mathrm{keV}$ for a single track of $600 \mathrm{MeV} / \mathrm{u}$ iron ion (see Fig 5b). The simulation results are also in qualitative agreement with experimental findings, which have reported that NMDA [5] and $\mathrm{GABA}_{\mathrm{A}}$ [19] receptors belong to the critical molecular targets in irradiated neural cells. These molecular structures play an important role in generation of action potential spikes, regulation of learning and memory in the CNS.

\subsection{Conclusion}

In conclusion, the Monte Carlo track structure simulations have been performed in order to analyze the energy transfer of charged particles produced in critical structures of the rat hippocampal neurons. In the first part of our study we have constructed hippocampal neurons from molecular targets to cell populations (see Fig 1, CA1 pyramidal neurons were selected for demonstration) by means of the Geant4/Geant4-DNA radiation transport codes. Thus, we should emphasize that the implementation of the realistic neuron morphology meets computational difficulties in dosimetric and electrical studies of large scale biological neural networks. For this reason, we have developed the geometry of the rat hippocampus on the basis of simplified neuron models with a smaller number of segments, but functional properties and dose deposition to be equivalent to realistic neuron morphologies. To demonstrate the opportunities of the proposed approach, we have implemented simple neuron geometries to 

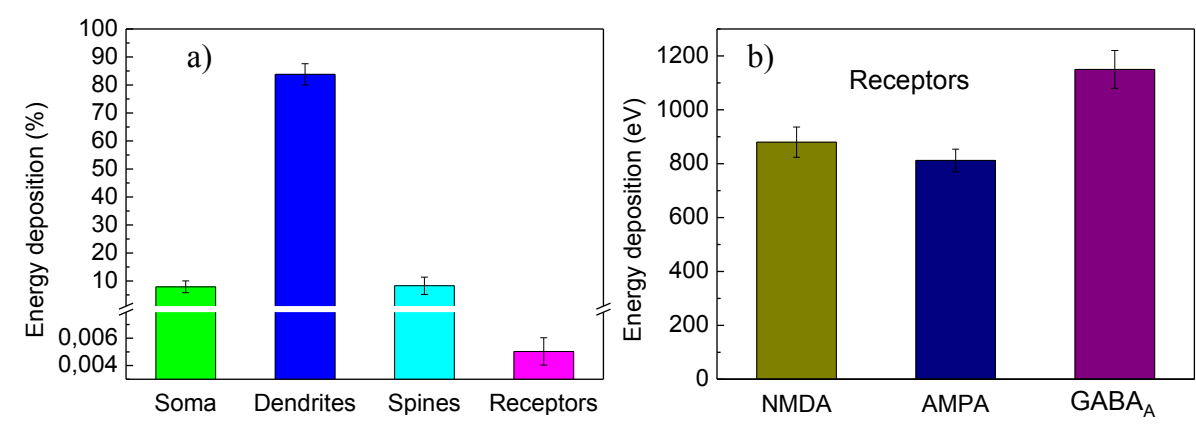

Figure 5. Percentage of energy deposition in different components (Soma, Dendrites, Spines and Receptors) of CA1 pyramidal neuron after irradiation (a). Energy deposition in different types of ionotropic receptors (NMDA, AMPA, $\mathrm{GABA}_{\mathrm{A}}$ ) per track of the iron ion with energy of $600 \mathrm{MeV} / \mathrm{u}$ (b)

construct the model of the rat hippocampus scaled about 1:100 with respect to a real structure, and computed the mean energy deposition in irradiated neurons of different types following the single track of $600 \mathrm{MeV} / \mathrm{u}$ iron ion (see Fig 2). Throughout the total number of 19367 neurons the most probable target has been presented by dense population of granular cells in DG region of hippocampus. The reported particle track simulations are both of fundamental and practical importance particularly in radiobiology for space radiation protection and radiation treatment of cancer cells.

Acknowledgements. The authors acknowledge the financial support from JINR AYSS (grant No.18-702-02) and from the Russian Foundation for Basic Research (grant No.17-2901007).

\section{References}

[1] I. El Naqa, P. Pater, J. Seuntjens, Phys. Med. Biol. 57, R75-R97 (2012)

[2] V.K. Parihar et al., Sci. Adv. 1, e1400256 (2005)

[3] P.H. Wu et al., PloS ONE. 7, e37677 (2012)

[4] R. Vlkolinsky et al., Radiat. Res. 173, 342 (2010)

[5] M. Machida, G. Lonart and R.A. Britten, Radiat. Res. 174618 (2010)

[6] D. Greene-Schloesser et al., Front. Oncol. 2, 73 (2012)

[7] A.O. Sapetsky et al., Biol. Bull. Rev. 7, 443 (2017)

[8] A.I. Grigoryev et al., Russ. J. Physiol. 99, 273 (2013)

[9] P. Andersen et al., The hippocampus book (Oxford University Press, NY, 2007) 100

[10] J. Allison et al., Nuclear Instruments and Methods A 835, 186-225 (2016)

[11] S. Incerti, et al., Med. Phys. 45, e722-e739 (2018)

[12] M.Batmunkh et al., Submitted to J.Phys.Med. (2018)

[13] L. Bayarchimeg et al., Mong. J. Phys. 2, 470-474 (2015)

[14] L. Bayarchimeg et al., Phys. Part. Nucl. Lett. 16, 54-62 (2019)

[15] M. Batmunkh et al., J. Radiat. Res. Appl. Sci. 8, 498-507 (2015)

[16] O. V. Belov, M. Batmunkh, S. Incerti et al., Physica Medica 32, 1510-1520 (2016)

[17] L. Bayarchimeg et al., EPJ WoC 173, 05005 (2017)

[18] M. Batmunkh et al., EPJ WoC 173, 05004 (2017)

[19] R. A. Britten et al., Radiat. Res. 182, 292-298 (2014) 